\title{
The delay with new-additive increase multiplicative decrease congestion avoidance and control algorithm.
}

\begin{abstract}
As the Internet becomes increasingly heterogeneous, the issue of congestion control becomes ever more important. And the queue length and end-to-end (congestion) delays are some of the important things in term of congestion avoidance and control mechanisms. In this research we continued to study the performances of the New-Additive Increase Multiplicative Decrease (AIMD) algorithm as one of the core protocols for TCP congestion avoidance and control mechanism, we want now to evaluate the effect of using the New-AIMD algorithm to measure the queue length and end-to-end delays and we will use the NCTUns simulator to get the results after make the modification of the mechanism. And we will use the Drop tail mechanism as Active Queue Management (AQM) in the bottleneck router. After implementation of our new approach with different number of flows, we will measure the delay for two types of delays (queuing delay and end-to-end delay), we expect the delay will be less with using our mechanism comparing with the mechanism in the previous study. Now and after got this results as low delay for bottleneck link case, we know the New-AIMD mechanism work as well under the network condition in the experiments.
\end{abstract}

Keyword: AIMD; Congestion control; End-to-end delay; Queue length; TCP; Queueing networks; Traffic congestion; Transmission control protocol. 